\title{
UNIFORM DECAY RATES OF SOLUTIONS TO A NONLINEAR WAVE EQUATION WITH BOUNDARY CONDITION OF MEMORY TYPE
}

\author{
Marcelo M. Cavalcanti* \\ Department of Mathematics - State University of Maringá \\ 87020-900, Maringá-PR, Brazil \\ mmcavalcanti@uem.br
}

Valéria N. Domingos Cavalcanti ${ }^{\dagger}$

Department of Mathematics - State University of Maringá 87020-900, Maringá - PR, Brazil

vndcavalcanti@uem.br

Mauro L. Santos

Departamento de Matemática, Universidade Federal do Pará

Campus Universitário do Guamá,

Rua Augusto Corrêa 01, Cep 66075-110, Pará, Brazil.

Is@ufpa.br

Abstract In this article we study the hyperbolic problem (1) where $\Omega$ is a bounded region in $\mathbf{R}^{n}$ whose boundary is partitioned into disjoint sets $\Gamma_{0}, \Gamma_{1}$. We prove that the dissipation given by the memory term is strong enough to assure exponential (or polynomial) decay provided the relaxation function also decays exponentially (or polynomially). In both cases the solution decays with the same rate of the relaxation function.

Keywords: wave equation, gradient nonlinearity, boundary memory term.

\footnotetext{
*Supported by CNPq(Brazil) - 301326/1996-7
}

†Supported by CNPq(Brazil) - 300567/1999-5 


\section{Introduction}

In this work we study the existence of global solutions and the asymptotic behavior of the energy related to the following nonlinear wave equation with a boundary condition of memory type

$$
\begin{aligned}
u_{t t}-\Delta u+F(x, t, u, \nabla u) & =0 \text { in } \Omega \times] 0,+\infty[ \\
u & \left.=0 \text { on } \Gamma_{0} \times\right] 0,+\infty[, \\
u+\int_{0}^{t} g(t-s) \frac{\partial u}{\partial \nu}(s) d s & \left.=0 \text { on } \Gamma_{1} \times\right] 0,+\infty[ \\
u(x, 0)=u^{0}(x), \quad u_{t}(x, 0) & =u^{1}(x) \text { in } \Omega,
\end{aligned}
$$

where $\Omega$ is a bounded domain of $\mathbf{R}^{n}, n \geq 1$, with smooth boundary $\Gamma=\Gamma_{0} \cup \Gamma_{1}$. Here, $\Gamma_{0}$ and $\Gamma_{1}$ are closed, disjoint, $\Gamma_{0} \neq \emptyset$ and $\nu$ is the unit normal vector pointing towards the exterior of $\Omega$. Equation (1c) is a nonlocal boundary condition responsible for the memory effect. Considering the history condition, we must add to conditions (1b)-(1c) the one given by

$$
\left.\left.u=0 \text { on } \Gamma_{0} \times\right]-\infty, 0\right] .
$$

We observe that in problem (1a)-(1d), $u$ represents the transverse displacement and the relaxation function $g$ is a positive non-increasing function belonging to $W^{2,1}(0,+\infty)$. Furthermore, suppose that the function $F: \bar{\Omega} \times\left[0,+\infty\left[\times \mathbf{R}^{n+1} \rightarrow \mathbf{R}\right.\right.$ is of class $C^{1}$ and satisfies

$$
|F(x, t, \xi, \zeta)| \leq C_{0}\left(1+|\xi|^{\gamma+1}+|\zeta|\right)
$$

where $C_{0}$ is a positive constant, and $\zeta=\left(\zeta_{1}, \ldots, \zeta_{n}\right)$.

Let $\gamma$ be a constant such that $\gamma>0$ for $n=1,2$, and $0<\gamma \leq 2 /(n-2)$ for $n \geq 3$. Assume that there is a non-negative function $\varphi(t)$ in the space $L^{\infty}(0, \infty) \cap L^{1}(0, \infty)$ such that

$$
F(x, t, \xi, \zeta) \eta \geq|\xi|^{\gamma} \xi \eta-\varphi(t)(1+|\eta \| \zeta|), \quad \forall \eta \in \mathbf{R},
$$

and, particularly,

$$
F(x, t, \xi, \zeta)(m \cdot \zeta) \geq|\xi|^{\gamma} \xi(m \cdot \zeta)-\varphi(t)(1+|\zeta||m \cdot \zeta|) .
$$

Consider the existence of positive constants $C_{0}, \ldots, C_{n}$, which verify

$$
\begin{gathered}
\left|F_{t}(x, t, \xi, \zeta)\right| \leq C_{0}\left(1+|\xi|^{\gamma+1}+|\zeta|\right) \\
\left|F_{\xi}(x, t, \xi, \zeta)\right| \leq C_{0}\left(1+|\xi|^{\gamma}\right) \\
\left|F_{\zeta_{i}}(x, t, \xi, \zeta)\right| \leq C_{i} \text { for } i=1,2, \ldots, n
\end{gathered}
$$


and also consider that there exist positive constants $D_{1}, D_{2}$, such that for all $\xi, \hat{\xi}, \eta, \hat{\eta} \in \mathbf{R}$ and for all $\zeta, \hat{\zeta} \in \mathbf{R}^{n}$,

$$
\begin{gathered}
(F(x, t, \xi, \zeta)-F(x, t, \hat{\xi}, \hat{\zeta}))(\eta-\hat{\eta}) \\
\geq-D_{1}\left(|\xi|^{\gamma}+|\hat{\xi}|^{\gamma}\right)|\xi-\hat{\xi}||\eta-\hat{\eta}|-D_{2}|\eta-\hat{\eta}||\zeta-\hat{\zeta}| .
\end{gathered}
$$

Defining

$$
F(x, t, u, \nabla u)=|u|^{\gamma} u+\varphi(t) \sum_{i=1}^{n} \sin \left(\frac{\partial u}{\partial x_{i}}\right),
$$

where $\varphi$ is a sufficiently regular function, we obtain an example of a function $F$ which verifies the above hypotheses.

In order to obtain the decay rates stated in theorems (2.1)-(2.2), we will assume that the function $\varphi(t)$ considered in (4) satisfies one of the following assumptions

$$
\varphi(t) \leq \theta_{0} e^{-b_{1} t} \quad \text { or } \quad \varphi(t) \leq \frac{\theta_{1}}{(1+t)^{p+1}},
$$

where $\theta_{0}, \theta_{1}$ and $b_{1}$ are positive constants and $p>1$.

The integral equation (1c) describes the memory effect which can be caused, for example, by the interaction with another viscoelastic element. Indeed, from the physical point of view, condition (1c) means that $\Omega$ is composed of a material which is clamped in a rigid body in $\Gamma_{0}$ and is clamped in a body with viscoelastic properties in the complementary part of its boundary named $\Gamma_{1}$. So, it is expected that if the kernel of the memory decays (exponentially or polynomially) the same occurs to the solutions of problem (1a) - (1d).

In what follows we are going to assume that there exists $x_{0} \in \mathbf{R}^{n}$ such that

$$
\begin{aligned}
& \Gamma_{0}=\left\{x \in \Gamma: \nu(x) \cdot\left(x-x_{0}\right) \leq 0\right\} \\
& \Gamma_{1}=\left\{x \in \Gamma: \nu(x) \cdot\left(x-x_{0}\right)>0\right\}
\end{aligned}
$$

Defining $m(x)=x-x_{0}$, the compactness of $\Gamma_{1}$ implies that there exists a positive constant $\delta_{0}$ such that

$$
0<\delta_{0} \leq m(x) \cdot \nu(x), \quad \forall x \in \Gamma_{1} .
$$

There is not much in literature regarding the existence and asymptotic behavior of evolution equations subject to memory conditions acting on the boundary. It is worth mentioning some papers in connection with viscoelastic effects on the boundary. In this direction we can cite the work 
by Aassila, Cavalcanti and Soriano [1] who considered the linear wave equation subject to nonlinear feedback and viscoelastic effects on the boundary, and proved uniform (exponential and algebraic) decay rates. Also, we can cite the article of Andrade and Munõz Rivera [2]where it was considered a one-dimensional nonlinear wave equation subject to a nonlocal and nonlinear boundary memory effect. In this work the authors showed that the dissipation occasioned by the memory term was strong enough to guarantee global estimates and, consequently, allowed them to prove existence of global smooth solution for small data and to obtain exponential(or polynomial) decay provided the kernel decays exponentially(or polynomially). In the same context we can mention the work of Santos [11], where decay rates were proved concerning the wave equation with coefficients depending on time and subject to a memory condition on the boundary.

A natural question that arises in this context is about the non-existence results for the wave equation in the presence of viscoelastic effects acting on the boundary. Concerning to this subject we can mention the work of Kirane and Tartar [6]who obtained non-existence results and Qin [9] who proved a blow up result for the nonlinear one dimensional wave equation with memory boundary condition.

In connection with the above discussion, regarding viscoelastic problems, it is important to cite the works of Ciarletta [4], Fabrizio and Morro [5] and Qin [8].

The main goal of the present paper is to complement the above mentioned works. The majority of the results are obtained in an one dimensional domain while our paper deals with a $n$-dimensional problem which brings up some additional difficulties, mainly in what concerns the geometric conditions. In addition, as we have a nonlinear problem whose nonlinearity $F=F(x, t, u, \nabla u)$ depends on the gradient, we do not have any information about the influence of the integral $\int_{\Omega} F(x, t, u, \nabla u) u_{t} d x$ on the energy $E(t)$ (see page 6) or about the sign of the derivative $E^{\prime}(t)$. In other words, we cannot guarantee that $E^{\prime}(t) \leq 0$ which plays an essential role in establishing the desired decay rates.

Note that condition (1b) implies that the solution of system (1a)-(1d) must belong to the following space

$$
V:=\left\{v \in H^{1}(\Omega): v=0 \text { on } \Gamma_{0}\right\} .
$$

The notations we use in this paper are standard and can be found in Lion's book [7]. In the sequel, $C$ (sometimes $C_{1}, C_{2}, \ldots$ ) is going to denote various positive constants which do not depend on $t$ and neither depend on the initial data. This paper is organized as follows. In section 2 we establish the existence and uniqueness for regular and weak 
solutions to the system(1a)-(1d). In section 3 we prove the uniform exponential decay and in section 4 we prove the uniform polynomial decay.

\section{Notations and Main Results}

In this section we present some notations and we are going to study the existence of regular and weak solutions to the system (1a)-(1d). First, we will use equation (1c) to estimate the term $\frac{\partial u}{\partial \nu}$.

Defining the convolution product operator by

$$
(g * \varphi)(t)=\int_{0}^{t} g(t-s) \varphi(s) d s
$$

and differentiating the equation (1c) with respect to $t$, we obtain the Volterra equation

$$
\left.\frac{\partial u}{\partial \nu}+\frac{1}{g(0)}\left(g^{\prime} * \frac{\partial u}{\partial \nu}\right)=-\frac{1}{g(0)} u_{t} \quad \text { on } \quad \Gamma_{1} \times\right] 0,+\infty[.
$$

Applying the Volterra's inverse operator, we get

$$
\frac{\partial u}{\partial \nu}=-\frac{1}{g(0)}\left(u_{t}+k * u_{t}\right) \quad \text { on } \quad \Gamma_{1} \times(0,+\infty) .
$$

where the resolvent kernel satisfies

$$
k+\frac{1}{g(0)} g^{\prime} * k=-\frac{1}{g(0)} g^{\prime} .
$$

Defining $\eta=\frac{1}{g(0)}$, we get

$$
\left.\frac{\partial u}{\partial \nu}=-\eta\left(u_{t}+k(0) u-k(t) u^{0}+k^{\prime} * u\right) \quad \text { on } \quad \Gamma_{1} \times\right] 0,+\infty[\text {. }
$$

Reciprocally, considering that the initial data satisfies $u^{0}=0$ on $\Gamma_{1}$, (11) implies (1c). Since we are interested in relaxation functions of exponential or polynomial type and identity (11) involves the resolvent kernel $k$, we want to investigate if $k$ has the same properties. The following Lemma answers this question.

Let $h$ be a relaxation function and $k$ its resolvent kernel, that is,

$$
k(t)-k * h(t)=h(t) .
$$

LEMMA 1 If $h$ is a positive continuous function, then $k$ is also a positive continuous function. Moreover,

1 If there exist positive constants $c_{0}$ and $\gamma$ with $c_{0}<\gamma$ such that

$$
h(t) \leq c_{0} e^{-\gamma t},
$$


we conclude that the function $k$ satisfies

$$
k(t) \leq \frac{c_{0}(\gamma-\epsilon)}{\gamma-\epsilon-c_{0}} e^{-\epsilon t},
$$

for all $0<\epsilon<\gamma-c_{0}$.

2 Let us consider $p>1$ and define by $c_{p}:=\sup _{t \in \mathrm{R}_{+}} \int_{0}^{t}(1+t)^{p}(1+$ $t-s)^{-p}(1+s)^{-p} d s$. Provided there exists a positive constant $c_{0}$ with $c_{0} c_{p}<1$ such that

$$
h(t) \leq c_{0}(1+t)^{-p},
$$

the function $k$ satisfies

$$
k(t) \leq \frac{c_{0}}{1-c_{0} c_{p}}(1+t)^{-p} .
$$

proof. Note that $k(0)=h(0)>0$. If we take $t_{0}=\inf \left\{t \in \mathbf{R}_{+}: k(t)=\right.$ $0\}$ we obtain that $k(t)>0$ for all $t \in\left[0, t_{0}\left[\right.\right.$. If $t_{0}<+\infty$, from equation (12) we get that $-k * h\left(t_{0}\right)=h\left(t_{0}\right)$ which is a contradiction. Therefore $k(t)>0$ for all $t \in\left[0,+\infty\left[\right.\right.$. Now, fixing $\epsilon$, such that $0<\epsilon<\gamma-c_{0}$ and defining

$$
k_{\epsilon}(t):=e^{\epsilon t} k(t), \quad h_{\epsilon}(t):=e^{\epsilon t} h(t),
$$

we get from (12) that $k_{\epsilon}(t)=h_{\epsilon}(t)+k_{\epsilon} * h_{\epsilon}(t)$. Hence

$$
\begin{aligned}
\sup _{s \in[0, t]} k_{\epsilon}(s) & \leq \sup _{s \in[0, t]} h_{\epsilon}(s)+\left(\int_{0}^{\infty} c_{0} e^{(\epsilon-\gamma) s} d s\right) \sup _{s \in[0, t]} k_{\epsilon}(s) \\
& \leq c_{0}+\frac{c_{0}}{(\gamma-\epsilon)} \sup _{s \in[0, t]} k_{\epsilon}(s)
\end{aligned}
$$

Therefore

$$
k_{\epsilon}(t) \leq \frac{c_{0}(\gamma-\epsilon)}{\gamma-\epsilon-c_{0}},
$$

which proves our first assertion. To show the second part let us introduce the following definitions

$$
k_{p}(t):=(1+t)^{p} k(t), \quad h_{p}(t):=(1+t)^{p} h(t) .
$$

Multiplying equation (12) by $(1+t)^{p}$ we obtain

$$
k_{p}(t)=h_{p}(t)+\int_{0}^{t} k_{p}(t-s)(1+t)^{p}(1+t-s)^{-p}(1+s)^{-p} h_{p}(s) d s
$$


and, consequently,

$$
\sup _{s \in[0, t]} k_{p}(s) \leq \sup _{s \in[0, t]} h_{p}(s)+c_{0} c_{p} \sup _{s \in[0, t]} k_{p}(s) \leq c_{0}+c_{0} c_{p} \sup _{s \in[0, t]} k_{p}(s),
$$

which implies

$$
k_{p}(t) \leq \frac{c_{0}}{1-c_{0} c_{p}} .
$$

This concludes the proof of Lemma.

Remark: In Racke [10, Lemma 7.4], it is assured that $c_{p}$ is a finite positive constant. Also, according to this Lemma, in what follows, we are going to use (11) instead of (1c).

In order to prove the following Lemma, let us define

$$
(g \diamond \varphi)(t):=\int_{0}^{t} g(t-s)|\varphi(t)-\varphi(s)|^{2} d s .
$$

LEMMA 2 For real functions $g, \varphi \in C^{1}([0, \infty[)$ we have

$$
(g * \varphi) \varphi_{t}=-\frac{1}{2} g(t)|\varphi(t)|^{2}+\frac{1}{2} g^{\prime} \diamond \varphi-\frac{1}{2} \frac{d}{d t}\left[g \diamond \varphi-\left(\int_{0}^{t} g(s) d s\right)|\varphi|^{2}\right] .
$$

The proof of this lemma follows by differentiating the term $g \diamond \varphi$.

The first order energy of system (1a)-(1d) is defined by

$$
\begin{aligned}
E(t):= & \frac{1}{2} \int_{\Omega}\left|u_{t}(x, t)\right|^{2} d x+\frac{1}{2} \int_{\Omega}|\nabla u(x, t)|^{2} d x \\
& +\frac{1}{\gamma+2} \int_{\Omega}|u(x, t)|^{\gamma+2} d x-\frac{\eta}{2}\left(k^{\prime} \diamond u\right)(t) \\
& +\frac{\eta}{2} k(t) \int_{\Gamma_{1}}|u(x, t)|^{2} d \Gamma .
\end{aligned}
$$

The well-posedness of system (1a)-(1d) as well as the decay rates expected are presented in the following Theorem.

THEOREM 3 Let $k \in W^{2,1}\left(\mathbf{R}_{+}\right)$, assume that assumptions (2)-(8) hold and suppose that $\left\{u^{0}, u^{1}\right\} \in\left(V \cap H^{2}(\Omega)\right)^{2}$, satisfying the compatibility condition

$$
\frac{\partial u^{0}}{\partial \nu}+\eta u^{1}=0 \quad \text { on } \quad \Gamma_{1} .
$$

Then, problem (1a)-(1d) possesses a unique solution $u$ such that $u \in L^{\infty}\left(0, \infty, V \cap H^{2}(\Omega)\right), u^{\prime} \in L^{\infty}(0, \infty, V), u^{\prime \prime} \in L^{\infty}(0, \infty, V)$. 
In addition, assuming that there exist positive constants $b_{1}, b_{2}$ which verify one of the conditions below

$$
k(0)>0, \quad k^{\prime}(t) \leq-b_{1} k(t), \quad k^{\prime \prime}(t) \geq-b_{2} k^{\prime}(t),
$$

or

$$
k(0)>0, \quad k^{\prime}(t) \leq-b_{1} k(t)^{1+\frac{1}{p}}, \quad k^{\prime \prime}(t) \geq b_{2}\left[-k^{\prime}(t)\right]^{1+\frac{1}{p+1}}, p>1
$$

and, moreover, that hypotheses (9)-(10) hold, we obtain that the energy $E(t)$ associated to problem (1a)-(1d) decays, respectively, with the following rates of decay

$$
E(t) \leq \alpha_{1} e^{-\alpha_{2} t} E(0)
$$

or

$$
E(t) \leq \frac{C}{(1+t)^{p+1}} E(0),
$$

where $\alpha_{1}, \alpha_{2}$ and $C$ are positive constants.

THEOREM 4 Let $k \in W^{2,1}\left(\mathbf{R}_{+}\right)$; suppose that $\left\{u^{0}, u^{1}\right\} \in V \times L^{2}(\Omega)$ and the assumptions (2)-(10) and (15)-(16) hold. Then, problem (1a)-(1d) has a unique weak solution $u$ in the space

$$
C^{0}([0, \infty) ; V) \cap C^{1}\left([0, \infty) ; L^{2}(\Omega)\right) .
$$

Furthermore, the decay rates presented in (17)-(18) hold for the weak solution $u$.

proof. The proof of existence and uniqueness for regular and weak solutions can be obtained following exactly identical procedure as in the work [3]of the authors Cavalcanti, Domingos Cavalcanti and Soriano. Consequently it will be omitted.

\section{Exponential Decay}

In this section we shall study the asymptotic behavior of the solutions of system (1a)-(1d) when the resolvent kernels $k$ is exponentially decreasing, that is, there exist positive constants $b_{1}, b_{2}$ such that

$$
k(0)>0, \quad k^{\prime}(t) \leq-b_{1} k(t), \quad k^{\prime \prime}(t) \geq-b_{2} k^{\prime}(t) .
$$

Note that this conditions implies that

$$
k(t) \leq k(0) e^{-b_{1} t} \quad \text { for } \quad t>0 .
$$


Our point of departure will be to establish some inequalities for the solution of system (1a)-(1d).

LEMMA 5 Any regular solution $u$ of the system (1a)-(1d) satisfy

$$
\begin{aligned}
\frac{d}{d t} E(t) \leq & -\frac{\eta}{2} \int_{\Gamma_{1}}\left|u_{t}\right|^{2} d \Gamma_{1}+\frac{\eta}{2} k^{2}(t) \int_{\Gamma_{1}}\left|u_{0}\right|^{2} d \Gamma_{1} \\
& +\frac{\eta}{2} k^{\prime}(t) \int_{\Gamma_{1}}|u|^{2} d \Gamma_{1}-\frac{\eta}{2} \int_{\Gamma_{1}} k^{\prime \prime} \diamond u d \Gamma_{1} \\
& +\varphi(t) \int_{\Omega}\left(1+\left|u_{t}\right||\nabla u|\right) d x .
\end{aligned}
$$

proof. Multiplying the equation (1a) by $u_{t}$ and integrating by parts over $\Omega$ we get

$$
\frac{1}{2} \frac{d}{d t} \int_{\Omega}\left(\left|u_{t}\right|^{2}+|\nabla u|^{2}\right) d x=-\int_{\Omega} F(x, t, u, \nabla u) u_{t} d x+\int_{\Gamma_{1}} \frac{\partial u}{\partial \nu} u_{t} d \Gamma_{1} .
$$

Taking (3), (11) into account and using Lemma 2 our conclusion follows.

Let us consider the following binary operator

$$
(k \diamond \varphi)(t):=\int_{0}^{t} k(t-s)(\varphi(t)-\varphi(s)) d s .
$$

Then employing Hölder's inequality for $0 \leq \mu \leq 1$ we have

$$
|(k \diamond \varphi)(t)|^{2} \leq\left[\int_{0}^{t}|k(s)|^{2(1-\mu)} d s\right]\left(|k|^{2 \mu} \diamond \varphi\right)(t) .
$$

Let us define the functionals

$$
\begin{aligned}
& \mathcal{N}(t):=\int_{\Omega}\left(\left|u_{t}\right|^{2}+|\nabla u|^{2}+|u|^{\gamma+2}\right) d x, \\
& \psi(t)=2 \int_{\Omega}(m \cdot \nabla u) u_{t}+\theta \int_{\Omega} u u_{t} d x,
\end{aligned}
$$

where $\theta \in] n-2, n\left[\right.$ and $\theta>\frac{2 n}{\gamma+2}$. The following Lemma plays an important role for the construction of the Lyapunov functional. 
LEMMA 6 For any regular solution of the system (1a)-(1d) we get

$$
\begin{aligned}
\frac{d}{d t} \psi(t) \leq & \int_{\Gamma_{1}}(m \cdot \nu)\left|u_{t}\right|^{2} d \Gamma_{1}+(\theta-n) \int_{\Omega}\left|u_{t}\right|^{2} d x \\
& -(\theta-(n-2)) \int_{\Omega}|\nabla u|^{2} d x \\
& +\int_{\Gamma_{1}} \frac{\partial u}{\partial \nu}\{m \cdot \nabla u+\theta u\} d \Gamma_{1}-\int_{\Gamma_{1}}(m \cdot \nu)|\nabla u|^{2} d \Gamma_{1} \\
& -\left(\theta-\frac{2 n}{\gamma+2}\right) \int_{\Omega}|u|^{\gamma+2} d x+\theta \varphi(t) \int_{\Omega}(1+|u||\nabla u|) d x \\
& +2 \varphi(t) \int_{\Omega}(1+|\nabla u||m \cdot \nabla u|) d x .
\end{aligned}
$$

proof. Differentiating the equation (21) with respect to $t$ and substituting the equation (1a) in the expression obtained we deduce

$$
\begin{aligned}
\frac{d}{d t} \psi(t)= & \int_{\Gamma_{1}}(m \cdot \nu)\left|u_{t}\right|^{2} d \Gamma_{1}+(\theta-n) \int_{\Omega}\left|u_{t}\right|^{2} d x \\
& +\int_{\Gamma_{1}} \frac{\partial u}{\partial \nu}\{m \cdot \nabla u+\theta u\} d \Gamma_{1} \\
& -\int_{\Gamma_{1}}(m \cdot \nu)|\nabla u|^{2} d \Gamma_{1}-(\theta-(n-2)) \int_{\Omega}|\nabla u|^{2} d x \\
& -2 \int_{\Omega} F(x, t, u, \nabla u) m \cdot \nabla u d x-\theta \int_{\Omega} F(x, t, u, \nabla u) u d x
\end{aligned}
$$

From the inequalities (3) and (4) we obtain

$$
\begin{aligned}
-\theta \int_{\Omega} F(x, t, u, \nabla u) u d x \leq & -\theta \int_{\Omega}|u|^{\gamma+2} d x \\
& +\theta \varphi(t) \int_{\Omega}(1+|u||\nabla u|) d x \\
-2 \int_{\Omega} F(x, t, u, \nabla u) m \cdot \nabla u d x \leq & -2 \int_{\Omega}|u|^{\gamma} u(m \cdot \nabla u) d x \\
& +2 \varphi(t) \int_{\Omega}(1+|\nabla u||m \cdot \nabla u|) d x .
\end{aligned}
$$

Substituting the inequalities (23)-(24) into (22) and noting that

$$
-\int_{\Gamma_{1}}(m \cdot \nu)|u|^{\gamma+2} d \Gamma_{1} \leq 0
$$

our conclusion follows. 
Decay Rates for a Nonlinear Wave Equation with Memory Type B.C.

To show that the energy decay exponentially we shall need of the following Lemma.

LEMMA 7 Let $f$ be a real positive function of class $C^{1}$. If there exists positive constants $\gamma_{0}, \gamma_{1}$ and $c_{0}$ such that

$$
f^{\prime}(t) \leq-\gamma_{0} f(t)+c_{0} e^{-\gamma_{1} t}
$$

then there exist positive constants $\gamma$ and $c$ such that

$$
f(t) \leq(f(0)+c) e^{-\gamma t} .
$$

proof. First, let us suppose that $\gamma_{0}<\gamma_{1}$. Define $F(t)$ by

$$
F(t):=f(t)+\frac{c_{0}}{\gamma_{1}-\gamma_{0}} e^{-\gamma_{1} t}
$$

Then

$$
F^{\prime}(t)=f^{\prime}(t)-\frac{\gamma_{1} c_{0}}{\gamma_{1}-\gamma_{0}} e^{-\gamma_{1} t} \leq-\gamma_{0} F(t) .
$$

Integrating above inequality over $] 0, t[$ we arrive to

$$
F(t) \leq F(0) e^{-\gamma_{0} t} \Rightarrow f(t) \leq\left(f(0)+\frac{c_{0}}{\gamma_{1}-\gamma_{0}}\right) e^{-\gamma_{0} t}
$$

Now, we shall assume that $\gamma_{0} \geq \gamma_{1}$. In this conditions we obtain

$$
f^{\prime}(t) \leq-\gamma_{1} f(t)+c_{0} e^{-\gamma_{1} t} \Rightarrow\left[e^{\gamma_{1} t} f(t)\right]^{\prime} \leq c_{0} .
$$

Integrating last expression over $] 0, t[$ we obtain

$$
f(t) \leq\left[f(0)+c_{0} t\right] e^{-\gamma_{1} t} .
$$

Since $t \leq\left(\gamma_{1}-\epsilon\right) e^{\left(\gamma_{1}-\epsilon\right) t}$ for any $0<\epsilon<\gamma_{1}$ we conclude that

$$
f(t) \leq\left[f(0)+c_{0}\left(\gamma_{1}-\epsilon\right)\right] e^{-\epsilon t} .
$$

This completes the proof.

Finally, we shall show the inequality (17) of the Teo 3. Using hypothesis (15) and Young inequality in Lemma 5 we get

$$
\begin{aligned}
\frac{d}{d t} E(t) \leq & -\frac{\eta}{2} \int_{\Gamma_{1}}\left(\left|u_{t}\right|^{2}-b_{2} k^{\prime} \diamond u+b_{1} k(t)|u|^{2}-\left|k(t) u_{0}\right|^{2}\right) d \Gamma_{1} \\
& +C_{\epsilon} \varphi^{2}(t)+\epsilon C \int_{\Omega}\left(\left|u_{t}\right|^{2}+|\nabla u|^{2}\right) d x,
\end{aligned}
$$


where $\epsilon$ is an arbitrary positive constant. Applying Young and Poincaré's inequalities in Lemma 6 we obtain

$$
\begin{aligned}
\frac{d}{d t} \psi(t) \leq & \int_{\Gamma_{1}}(m \cdot \nu)\left|u_{t}\right|^{2} d \gamma_{1}+(\theta-n) \int_{\Omega}\left|u_{t}\right|^{2} d x \\
& -(\theta-(n-2)) \int_{\Omega}|\nabla u|^{2} d x \\
& -\left(\theta-\frac{2 n}{\gamma+2}\right) \int_{\Omega}|u|^{\gamma+2} d x+C_{\epsilon} \varphi^{2}(t) \\
& +\epsilon C\left\{\int_{\Gamma_{1}}(m \cdot \nu)|\nabla u|^{2} d \Gamma_{1}+\mathcal{N}(t)\right\}+C_{\epsilon} \int_{\Gamma_{1}}\left|\frac{\partial u}{\partial \nu}\right|^{2} d \Gamma_{1} \\
& -\int_{\Gamma_{1}}(m \cdot \nu)|\nabla u|^{2} d \Gamma_{1} .
\end{aligned}
$$

Noting that the boundary condition (11) can be written as $\frac{\partial u}{\partial \nu}=$ $-\eta\left\{u_{t}+k(t) u-k^{\prime} \diamond u-k(t) u_{0}\right\}$ we arrive at

$$
\begin{aligned}
\frac{d}{d t} \psi(t) \leq & (\theta-n) \int_{\Omega}\left|u_{t}\right|^{2} d x-(\theta-(n-2)) \int_{\Omega}|\nabla u|^{2} d x \\
& -\left(\theta-\frac{2 n}{\gamma+2}\right) \int_{\Omega}|u|^{\gamma+2} d x \\
& -\int_{\Gamma_{1}}(m \cdot \nu)|\nabla u|^{2} d \Gamma_{1}+C_{\epsilon} \varphi^{2}(t) \\
& +\epsilon C\left\{\int_{\Gamma_{1}}(m \cdot \nu)|\nabla u|^{2} d \Gamma_{1}+\mathcal{N}(t)\right\} \\
& +C_{\epsilon} \int_{\Gamma_{1}}\left(\left|u_{t}\right|^{2}+|k(t) u|^{2}+\left|k^{\prime} \diamond u\right|^{2}+\left|k(t) u_{0}\right|^{2}\right) d \Gamma_{1} .
\end{aligned}
$$

On the other hand applying the inequality (20) with $\mu=\frac{1}{2}$ in inequality (26) we obtain

$$
\begin{aligned}
\frac{d}{d t} \psi(t) \leq & (\theta-n) \int_{\Omega}\left|u_{t}\right|^{2} d x-(\theta-(n-2)) \int_{\Omega}|\nabla u|^{2} d x \\
& -\left(\theta-\frac{2 n}{\gamma+2}\right) \int_{\Omega}|u|^{\gamma+2} d x \\
& -\int_{\Gamma_{1}}(m \cdot \nu)|\nabla u|^{2} d \Gamma_{1}+C_{\epsilon} \varphi^{2}(t) \\
& +\epsilon C\left\{\int_{\Gamma_{1}}(m \cdot \nu)|\nabla u|^{2} d \Gamma_{1}+\mathcal{N}(t)\right\} \\
& +C_{\epsilon} \int_{\Gamma_{1}}\left(\left|u_{t}\right|^{2}+k(t)|u|^{2}-k^{\prime} \diamond u+\left|k(t) u_{0}\right|^{2}\right) d \Gamma_{1} .
\end{aligned}
$$


Let us introduce the Lyapunov functional

$$
\mathcal{L}(t):=N E(t)+\psi(t),
$$

with $N>0$. Taking $N$ large and $\epsilon$ small enough, the previous inequalities imply that

$$
\frac{d}{d t} \mathcal{L}(t) \leq-C_{0} E(t)+C_{1} R^{2}(t) E(0)
$$

where $R(t)=k(t)+\varphi(t)$. Moreover, using Young's inequality and taking $N$ sufficiently large we find that

$$
q_{0} E(t) \leq \mathcal{L}(t) \leq q_{1} E(t),
$$

for some positive constants $q_{0}$ and $q_{1}$. From this inequality we conclude that

$$
\frac{d}{d t} \mathcal{L}(t) \leq-\frac{C_{0}}{q_{1}} \mathcal{L}(t)+C_{1} R^{2}(t) E(0)
$$

which implies, in view of Lemma 7 and from the exponential decay of $k$, $\varphi$, that

$$
\mathcal{L}(t) \leq\{\mathcal{L}(0)+C\} e^{-\alpha_{2} t},
$$

for some positive constants $C, \alpha_{2}$. From the inequality (29) our conclusion follows.

\section{Polynomial Rate of Decay}

Here our attention will be focused on the uniform decay rate when the resolvent kernel $k$ decays polynomially like $(1+t)^{-p}$. In this case we will show that the solution also decays polynomially with the same rate. Therefore, we will assume that the resolvent kernel $k$ satisfies

$$
k(0)>0, \quad k^{\prime}(t) \leq-b_{1} k(t)^{1+\frac{1}{p}}, \quad k^{\prime \prime}(t) \geq b_{2}\left[-k^{\prime}(t)\right]^{1+\frac{1}{p+1}}
$$

for some $p>1$ and some positive constants $b_{1}$ and $b_{2}$.

The lemmas below will play an important role in the sequel.

LEMMA 8 Let $u$ be a solution of system (1a)-(1d). Then, for $p>1$, $0<r<1$ and $t \geq 0$, we have

$$
\begin{gathered}
\left(\int_{\Gamma_{1}}\left|k^{\prime}\right| \diamond u d \Gamma_{1}\right)^{\frac{1+(1-r)(p+1)}{(1-r)(p+1)}} \\
\leq\left(2 \int_{0}^{t}\left|k^{\prime}(s)\right|^{r} d s \|\left. u\right|_{L^{\infty}\left(0, t_{i} L^{2}\left(\Gamma_{1}\right)\right)} ^{2}\right)^{\frac{1}{(1-r)(p+1)}} \int_{\Gamma_{1}}\left|k^{\prime}\right|^{1+\frac{1}{p+1}} \diamond u d \Gamma_{1}
\end{gathered}
$$


while for $r=0$ we get

$$
\begin{gathered}
\left(\int_{\Gamma 1}\left|k^{\prime}\right| \diamond u d \Gamma_{1}\right)^{\frac{p+2}{p+1}} \\
\leq 2\left(\int_{0}^{t}\|u(s, .)\|_{L^{2}\left(\Gamma_{1}\right)}^{2} d s+\left.t|| u(s, .)\right|_{L^{2}\left(\Gamma_{1}\right)} ^{2}\right)^{p+1} \int_{\Gamma_{1}}\left|k^{\prime}\right|^{1+\frac{1}{p+1}} \diamond u d \Gamma_{1} .
\end{gathered}
$$

proof. See e. g. [12]

LEMMA 9 Let $f \geq 0$ be a differentiable function satisfying

$$
f^{\prime}(t) \leq-\frac{c_{1}}{f(0)^{\frac{1}{\alpha}}} f(t)^{1+\frac{1}{\alpha}}+\frac{c_{2}}{(1+t)^{\beta}} f(0) \quad \text { for } t \geq 0
$$

for some positive constants $c_{1}, c_{2}, \alpha$ and $\beta$ such that

$$
\beta \geq \alpha+1 \text {. }
$$

Then there exists a constant $c>0$ such that

$$
f(t) \leq \frac{c}{(1+t)^{\alpha}} f(0) \quad \text { for } \quad t \geq 0
$$

proof. See e. g. [11]

Finally, we shall prove the inequality (18). Using hypothesis (30) in Lemma 5 yields

$$
\begin{aligned}
E^{\prime}(t) & \leq-\frac{\eta}{2} \int_{\Gamma_{1}}\left(\left|u_{t}\right|^{2}+b_{2}\left[-k^{\prime}\right]^{1+\frac{1}{p+1}} \diamond u+b_{1} k^{1+\frac{1}{p}}(t)|u|^{2}-\left|k(t) u_{0}\right|^{2}\right) d \Gamma_{1} \\
& +C_{\epsilon} \varphi^{2}(t)+\epsilon C \int_{\Omega}\left(\left|u_{t}\right|^{2}+|\nabla u|^{2}\right) d x .
\end{aligned}
$$

Considering inequality (20) with $\mu=\frac{p+2}{2(p+1)}$ and taking hypothesis (30) into account we obtain the estimate

$$
\left|k^{\prime} \diamond u\right|^{2} \leq C\left[-k^{\prime}\right]^{1+\frac{1}{p+1}} \diamond u
$$

Using the above inequalities in Lemma 6 , yields

$$
\begin{aligned}
\frac{d}{d t} \psi(t) \leq & +(\theta-n) \int_{\Omega}\left|u_{t}\right|^{2} d x-(\theta-(n-2)) \int_{\Omega}|\nabla u|^{2} d x \\
& -\left(\theta-\frac{2 n}{\gamma+2}\right) \int_{\Omega}|u|^{\gamma+2} d x \\
& -\int_{\Gamma_{1}}(m \cdot \nu)|\nabla u|^{2} d \Gamma_{1} \\
& +C_{\epsilon} \varphi^{2}(t)+\epsilon C\left\{\int_{\Gamma_{1}}(m \cdot \nu)|\nabla u|^{2} d \Gamma_{1}+\mathcal{N}(t)\right\} \\
& +C \int_{\Gamma_{1}}\left(\left|u_{t}\right|^{2}+k^{1+\frac{1}{p}}(t)|u|^{2}+\left[-k^{\prime}\right]^{1+\frac{1}{p+1}} \diamond u+\left|k(t) u_{0}\right|^{2}\right) d \Gamma_{1}
\end{aligned}
$$


In this conditions, taking $N$ sufficiently large and $\epsilon$ small enough the Lyapunov functional defined in (28) satisfies

$$
\begin{aligned}
\frac{d}{d t} \mathcal{L}(t) \leq & -C_{0} \mathcal{N}(t)+C_{1} R^{2}(t) E(0) \\
& -C_{2} \int_{\Gamma_{1}}\left[-k^{\prime}\right]^{1+\frac{1}{p+1}} \diamond u d \Gamma_{1} .
\end{aligned}
$$

Let us fix $0<r<1$ such that $\frac{1}{p+1}<r<\frac{p}{p+1}$. From (30) we have that

$$
\int_{0}^{\infty}\left|k^{\prime}\right|^{r} \leq C \int_{0}^{\infty} \frac{1}{(1+t)^{r(p+1)}}<\infty .
$$

Using this estimate in Lemma 9 we get

$$
\begin{array}{r}
\int_{\Gamma_{1}}\left[-k^{\prime}\right]^{1+\frac{1}{p+1}} \diamond u d \Gamma_{1} \\
\geq C E(0)^{-\frac{1}{(1-r)(p+1)}}\left(\int_{\Gamma_{1}}\left[-k^{\prime}\right] \diamond u d \Gamma_{1}\right)^{1+\frac{1}{(1-r)(p+1)}} .
\end{array}
$$

On the other hand, from the Trace theorem we deduce

$$
E(t)^{1+\frac{1}{(1-r)(p+1)}} \leq C E(0)^{\frac{1}{(1-r)(p+1)}} \mathcal{N}(t) .
$$

Substituting (32)-(33) into (30) we obtain

$$
\begin{aligned}
\frac{d}{d t} \mathcal{L}(t) \leq & -C E(0)^{-\frac{1}{(1-r)(p+1)}} E(t)^{1+\frac{1}{(1-r)(p+1)}}+C_{1} R^{2}(t) E(0) \\
& -C E(0)^{-\frac{1}{(1-r)(p+1)}}\left(\int_{\Gamma_{1}}\left[-k^{\prime}\right] \diamond u d \Gamma_{1}\right)^{1+\frac{1}{(1-r)(p+1)}}
\end{aligned}
$$

Taking into account the inequality (29) we conclude that

$$
\frac{d}{d t} \mathcal{L}(t) \leq-\frac{C}{\mathcal{L}(0)^{\frac{1}{(1-r)(p+1)}}} \mathcal{L}(t)^{1+\frac{1}{(1-r)(p+1)}}+C_{1} R^{2}(t) E(0),
$$

which implies, applying Lemma 9, that

$$
\mathcal{L}(t) \leq \frac{C}{(1+t)^{(1-r)(p+1)}} \mathcal{L}(0) .
$$

Since $(1-r)(p+1)>1$ we get, for $t \geq 0$, the following bounds

$$
\begin{aligned}
t\|u\|_{L^{2}\left(\Gamma_{1}\right)}^{2} \leq t \mathcal{L}(t) & <\infty, \\
\int_{0}^{t}\|u\|_{L^{2}\left(\Gamma_{1}\right)}^{2} d s \leq C \int_{0}^{t} \mathcal{L}(s) d s & <\infty .
\end{aligned}
$$


Considering the above estimates in Lemma 9 with $r=0$ it holds that

$$
\int_{\Gamma_{1}}\left[-k^{\prime}\right]^{1+\frac{1}{p+1}} \diamond u d \Gamma_{1} \geq \frac{c}{E(0)^{\frac{1}{p+1}}}\left(\int_{\Gamma_{1}}\left[-k^{\prime}\right] \diamond u d \Gamma\right)^{1+\frac{1}{p+1}} .
$$

Using the last inequality instead of (32) and reasoning in the same way as above we conclude that

$$
\frac{d}{d t} \mathcal{L}(t) \leq-\frac{c}{\mathcal{L}(0)^{\frac{1}{p+1}}} \mathcal{L}(t)^{1+\frac{1}{p+1}}+C_{1} R^{2}(t) E(0) .
$$

Applying Lemma 9 again, we obtain

$$
\mathcal{L}(t) \leq \frac{c}{(1+t)^{p+1}} \mathcal{L}(0)
$$

Finally, from (29) we conclude

$$
E(t) \leq \frac{c}{(1+t)^{p+1}} E(0),
$$

which completes the proof.

\section{Acknowledgments}

The authors wish to thank Irena Lasiecka and all the organizers for their kind attention and the nice moments during the 21 st IFIP.

\section{References}

[1] M. Aassila, M. M. Cavalcanti, and J. A. Soriano. Asymptotic stability and energy decay rates for solutions of the wave equation with memory in a starshaped domain. SIAM J. Control Optim., 38(5):1581-1602, 2000.

[2] D. Andrade and J. E. Muñoz Rivera. Exponential decay of non-linear wave equation with viscoelastic boundary condition. Math. Meth. Appl. Sci, 23:41$61,2000$.

[3] M. M. Cavalcanti, V. N. Domingos Cavalcanti, and J. A. Soriano. Existence and boundary stabilization of a nonlinear hyperbolic equation with time-dependent coefficients. Electron. J. Differential Equations, 1998(08):1-21, 1998.

[4] M. Ciarletta. A differential problem for the heat equation with a boundary condition with memory. Appl. Math. Lett., 10(1):95-101, 1997.

[5] M. Fabrizio and A. Morro. A boundary condition with memory in electromagnetism. Arch. Rational Mech. Anal., 136:359-381, 1996.

[6] M. Kirane and N. Tartar. Non-existence results for a semilinear hyperbolic problem with boundary condition of memory type. Journal for Analysis and Its Applications, 19(2):453-468, 2000. 
[7] J. L. Lions. Quelques Mèthodes de resolution de problèmes aux limites non lineaires. Dunod Gauthiers Villars, Paris, 1969.

[8] T. Qin. Global solvability of nonlinear wave equation with a viscoelastic boundary condition. Chin. Ann. Math., 14B(3):335-346, 1993.

[9] T. Qin. Breakdown of solutions to nonlinear wave equations with a viscoelastic boundary condition. Arab. J. Sci. Engng., 19(2A):195-201, 1994.

[10] R. Racke. Lectures on nonlinear evolution equations. Initial value problems. Aspect of Mathematics E19. Friedr. Vieweg \& Sohn, Braunschweig, Wiesbaden, 1992.

[11] M. L. Santos. Decay rates for solutions of a system of wave equations with memory. E. J. Diff. Eqs., 2002(38):1-17.

[12] M. L. Santos. Asymptotic behavior of solutions to wave equations with a memory condition at the boundary. E. J. Diff. Eqs., 2001(73):1-11. 